\title{
Polychromatic Sets Theory and its Application in Enterprise Information Systems
}

\author{
Zongbin $\mathrm{Li}^{i}$, Lida $\mathrm{Xu}^{2}$, and Shanshan Zhao ${ }^{1}$ \\ 1.State Key Laboratory for Manufacturing Systems Engineering, \\ College of Mechanical Engineering, Xi'an Jiaoong University, Xi'an \\ 710049, China \\ Lzb9910@mail.xjtu.edu.cn, shanshanzhao@stu.xjtu.edu.cn \\ 2.Depatment of Information Technology and Decision Science, \\ Old Dominion University, Norfolk, VA 23529, USA \\ LXu@odu.edu
}

\begin{abstract}
Firstly, the basic conception of polychromatic sets, polychromatic graph and operation of the polychromatic sets and polychromatic graph is proposed. Secondly, the our main research status of polychromatic sets in enterprises are detailed, which includes the application of the polychromatic sets in the conceptual design, the application of the polychromatic sets in the product assembly modeling, the application of the polychromatic sets in the work flow modeling, the application of the polychromatic sets in tolerance modeling. Finally, the research conclusion and foreground is introduced.
\end{abstract}

\section{Introduction}

Enterprise information technology is the important weapon to improve and keep an enterprises' competitiveness in ever-changing business environment. It is a systematic methodology that is mostly required as a supporting tool achieving complicated activities connected with introduction of enterprise information systems. The enterprise information systems embodied to be impertinent can be wasting enterprise resources and weakening enterprise's competitiveness. Therefore, many consulting corporations have developed and applied various commercial methodologies in order to offer systematic guide on a construction of enterprise information systems. Methodology integrates each kinds of theory and tools scattered and must support that all of the users may utilize it easily. Thus, related methodology research must connect each kind of theory and tools in synthetic

Please use the following format when citing this chapter:

Li, Z., Xu, L., Zhao, S., 2006, in International Federation for Information

Processing, Volume 205, Rescarch and Practical Issucs of Enterprise Information Systems, cds.

Tjoa, A.M., Xu, L., Chaudhry, S., (Boston:Springer), pp.721-728. 
viewpoint to satisfy efficient construction efficiency and effectiveness of information systems. Also, previous researches show that enterprises that have systematic methodology construct more effectively information systems. More research works and commercial products, however, are lack of the architectural integrity and functional applicability to meet these sophisticated needs of enterprises. Lack of the architectural integrity is caused by two factors: absence of customizable architecture regarding inner environment and natural culture of enterprises, and non-integrated framework to manage engineering tools and output data used and generated during development and implementation of information systems. Lack of the functional applicability is caused by three factors: broken bridge linking business strategy with information strategy in rational manner, absence of economic justification and management systems, and unreliable mechanism for analysis and evaluation about level of enterprise information systems [1].

In Russia in the last century, professor V.V. Pavlov, who is the famous expert of aeronautical technology design of Russia, suggested the conception of polychromatic graph in 1988[2], presented the concept of polychromatic sets in 1995[3] and proposed the polychromatic sets theory in 2002[4], through accumulation, generalization and abstraction. Now the theory has become the major direction in Russia, and the systematic theory and technique has been widely used in enterprises of Russia, especially in the aerospace and aviation enterprises. The new methodology introduced in this paper can assure architectural integrity and functional applicability for successful development and implementation of information systems.

\subsection{The basic conception of polychromatic sets}

The composition of a conventional set $\mathrm{A}$ is as follows:

$$
A=\left(a_{1}, a_{2}, \cdots, a_{n}\right)
$$

For the elements of $a_{i}, a_{j} \in A$, the difference resides in their names even though these elements could be different. It is impossible to represent all other characteristics in the conventional set. In polychromatic sets, not only its elements, but also its entirety can be pigmented with different colors to represent the research object as well as the properties of its elements. The elements of conventional sets are also elements of the polychromatic sets. The color set $\mathrm{F}\left(a_{i}\right)$ corresponds to every element $a_{i} \in \mathrm{A}$ and the color set $\mathrm{F}(\mathrm{A})$ corresponds to the entirety of $\mathrm{A}$,

$$
\begin{array}{r}
F(A)=\left(F_{1}(A), F_{2}(A), \cdots, F_{n}(A)\right) \\
F\left(a_{i}\right)=\left(F_{1}\left(a_{i}\right), F_{2}\left(a_{i}\right), \cdots, F_{m_{i}}\left(a_{i}\right)\right)
\end{array}
$$

The color constituents of polychromatic set $F(A)$ and the color constituents of its elements $F\left(a_{i}\right)$ are called pigmentation $\square$ they are included in a unified color set,

$$
\begin{aligned}
& F \supseteq F(A) ; F\left(a_{i}\right), \mathrm{i}=1,2, \ldots, n \\
& F(a)=\bigcup_{i=1}^{n} F\left(a_{i}\right)
\end{aligned}
$$


$F(A) \neq F(a)$, obviously, the characteristics of the sets is not the total of the characteristics of the element. If an object is represented in terms of polychromatic sets, its color $F_{j}(A)$ and $F_{j}\left(a_{i}\right)$, the color of the element $a_{i}$, correspond to $j$ th characteristics of the object or the element.

In the polychromatic sets theory, the relationship between element and unified color can be represented using the following Boolean matrix $[\mathrm{A} \times \mathrm{F}(\mathrm{A})]$.

$$
\left\|c_{i(j)}\right\|_{A, F(a)}=[A \times F(A)]=\left[\begin{array}{ccccc}
F_{1} & \ldots & F_{j} & \ldots & F_{m} \\
c_{1(1)} & \ldots & c_{1(j)} & \ldots & c_{1(m)} \\
\ldots & \ldots & \ldots & \ldots & \ldots \\
c_{i(1)} & \ldots & c_{i(j)} & \ldots & c_{i(m)} \\
\ldots & \ldots & \ldots & \ldots & \ldots \\
c_{k(1)} & \ldots & c_{k(j)} & \ldots & c_{k(m)}
\end{array}\right] \begin{gathered}
a_{1} \\
\ldots \\
a_{i} \\
\ldots \\
a_{k}
\end{gathered}
$$

In which $c_{i(j)}=1$, if $F_{j} \square F\left(a_{i}\right)$, and $F(a)=\bigvee_{i=1}^{n} F\left(a_{i}\right)$

The relationship between individual color and unified color can be represented using the following Boolean matrix $[\mathrm{F}(\mathrm{a}) \times \mathrm{F}(\mathrm{A})]$.

$$
\left\|c_{i(j)}\right\|_{F(a) . F(A)}=[F(a) \times F(A)]=\left[\begin{array}{ccccc}
F_{1} & \ldots & F_{j} & \ldots & F_{m} \\
c_{1(1)} & \ldots & c_{i(j)} & \ldots & c_{1(m)} \\
\ldots & \ldots & \ldots & \ldots & \ldots \\
c_{i(1)} & \ldots & c_{i(j)} & \ldots & c_{i(m)} \\
\ldots & \ldots & \ldots & \ldots & \ldots \\
c_{k(1)} & \ldots & c_{k(j)} & \ldots & c_{k(m)}
\end{array}\right] \begin{aligned}
& f_{1} \\
& \ldots \\
& f_{i} \\
& f_{k}
\end{aligned}
$$

In which $c_{i(j)}=1$, assuming individual color $f_{i}$ affects the existence of unified color $F_{j}$.

\subsection{The basic conception of polychromatic graph}

The composition of polychromatic graph is $P G=\left(F(G), P S_{A}, P S_{C}\right)$.

$$
P S_{A}=(A, F(a), F(A),[A \times F(a)],[A \times F(A)],[A \times A(F)]
$$

represents the nodes of the polychromatic graph. A represents the nodes; $F(a)$ represents the individual color of the element; $F(A)$ represents the unified color; $A \times F(a)$ represents the relationship between the elements and individual color; $A \times F(A)$ represents the relationship between the elements and unified color; $A \times A(F)$ represents the entity of unified color.

$$
P S_{C}=(C, F(c), F(C),[C \times F(c)],[C \times F(C)],[C \times C(F)]
$$

represents the polychromatic sets of the borders, in which $F(G)$ represents the unified color. The Disjunction polychromatic sets and the Conjunction polychromatic sets are built based on the disjunction algorithms and conjunction 
algorithms of polychromatic sets. If the borders of polychromatic graph has no colors, $F(G)=F(A) \cup F(C)=F(A)$; if both the borders and nodes are no colors, this polychromatic graph is the normal graph.

If each node is the pigmentation of one color, only one element in the $i$ th row of the Boolean matrix $[F(a) \times F(A)]$ is $1\left(c_{i(j)}=1\right)$. If the Boolean matrixes of borders have the same character, and the nodes and borders of this polychromatic graph have only color, this polychromatic graph is called unicolor graph.

If the nodes of the polychromatic graph can be pigmentized several colors (if the nodes of the polychromatic graph are the pigmentation of several colors), several elements are in the $i$ th row of the Boolean matrix $[F(a) \times F(A)]$ is $1\left(c_{i(j 1)}=c_{i(j 2)}=\cdots=c_{i(j n)}=1\right)$, and this nodes are polychrome, the borders of the graph possible have the same character, so this graph is called polychromatic graph.

\subsection{The operation of the polychromatic sets and polychromatic graph}

In the polychromatic graph, consider the restrict condition of the nodes and borders; build the map relation between the colors and elements of the object. In the polychromatic sets, a set of nodes (borders) is called nodes set (borders set), $S_{i}=\left(a_{i_{1}}, a_{i_{2}}, \cdots, a_{i_{n}}\right)$, in which $S_{i} \subseteq A, a_{i k} \in A$. In the polychromatic sets, the pigmented functions of nodes set is $F\left(S_{i}\right)_{A}=R\left(F\left(a_{i_{1}}\right), F\left(a_{i_{2}}\right), \cdots, F\left(a_{i_{n}}\right)\right)$, the pigmented functions of borders set is $F\left(S_{i}\right)_{C}=R\left(F\left(c_{i_{1}}\right), F\left(c_{i_{2}}\right), \cdots, F\left(c_{i_{n}}\right)\right)$, so $F\left(S_{i}\right)=R\left(F\left(S_{i}\right)_{A}, F\left(S_{i}\right)_{C}\right)$. The operation of the polychromatic graph is different from the normal graph. In the polychromatic graph, the algorithms of the pigmented functions are as follows:

(1) Conjunction and Disjunction

The relationships between the colors include conjunction and disjunction. Firstly the vector space $F=\left(F_{1}, F_{2}, \cdots, F_{n}\right)$ is introduced in which $(F(A), F(C) \subseteq F)$; the logical relation is $F\left(S_{i}\right)_{A}=\underset{k=1}{\vee} F\left(a_{i_{k}}\right), F\left(S_{i}\right)_{C}={ }_{k=1}^{n-1} F\left(c_{i_{k}\left(i_{k+1}\right)}\right), F\left(S_{i}\right)=F\left(S_{i}\right)_{A} \vee F\left(S_{i}\right)_{C}$.colors matrix is called conjunction In the operations of polychromatic sets, the conjunction relation between colors is $F\left(S_{i}\right)_{A}=\wedge_{k=1}^{n} F\left(a_{i_{k}}\right), F\left(S_{i}\right)_{C}=\wedge_{k=1}^{n-1} F\left(c_{i_{k}\left(i_{k+1}\right)}\right), F\left(S_{i}\right)=F\left(S_{i}\right)_{A} \wedge F\left(S_{i}\right)_{C}$.

(2) The entity operation of the polychromatic sets

If the element $a_{i} \in S$ and the $F_{j}(S)$ are exist, the entity of the unified colors $F_{j}$ is $S_{k}\left(F_{j}\right)=\left(a_{i 1}, a_{i 2}, \cdots, a_{i n}\right)$ which the Boolean matrix represents $\left\|c_{i(j)}\right\|_{A, A(F)}=[S \times S(F)]$. The all entity elements of the all unified colors 
comprise $S \times S(F)$, in which the $S(F)$ is the combination of the all entities of the all unified colors; the individual entity relate to not only the individual unified colors, but also the achieved colors group. According to the definition of polychromatic sets, each the each entity group can achieve entity colors. For the polychromatic sets, each entity has one or several members. in the whole Boolean space of the polychromatic sets, the result sets which satisfying its polychromatic sets can be gained by operating the members according to the conjunction and disjunction of polychromatic sets.

(3) Descartes product

Suppose A and B are two random sets, the elements in the A are the first elements and the elements in B are the second elements, so the ordinal combinations are built. All ordinal combinations are called the Descartes product of the A, B, recorded as $\mathrm{A} \times \mathrm{B}[5,6]$.

\section{The research status of polychromatic sets}

\subsection{Application of the polychromatic sets in the conceptual design}

Conceptual design is the process of achieving plans by studying out the function structure, seeking the proper function principle and choosing the basic approach through abstraction after deciding the assignment and the search algorithm can be built by using fuzzy mathematical model [7]. The key technology of Computer Aided Conceptual design comprises product information modeling and the technology of seeking schemes and so on [8]

Currently product information modeling of Conceptual design, such as the function method tree, function tree and function structure graph, must use geometrical graph, so it is difficult to be recognized with computers, furthermore not express the knowledge completely. Our team proposes the method of using the layered Hierarchy-Tree-Model of polychromatic sets to formalize the product information model express each layer function of the product as the unified colors and the individual colors and build the reason matrix and restriction matrix of the unified colors and the individual colors to provide the facility for the expression and operation of the computer [9-16]. Search the reason matrix and restriction matrix layer by layer to exclude the illegitimate scheme, and achieve the process of searching the reason matrix and restriction matrix from the whole function to the feasible scheme, so it offers facility for Computer Aided Conceptual design [17].

\subsection{Application of the polychromatic sets in the product assembly modeling}

The automatic generation of assembly sequence is the key link of computer aided assembles process planning (CAAPP). In the process of automatic generation of assembly sequence, the primary question is the representation of the assembly 
information and the building of the assembly relation model. At present, the expanded AND/OR graph, directed graph and Petri nets are widely used to build the assembly relation model, but there are many disadvantages: (1) the structures are complicated. (2) exist "combinatorial explosion ". (3) difficult to simulate the largescale and complex systems, and (4) not facilitated to realize the system [18].

By using polychromatic sets, the combinations of each two parts of the assembly body are looked upon as the elements of polychromatic sets, the locating relationships between the two parts are considered as the holistic contours of polychromatic sets, the locating datum model can be built; the combinations of each two parts of the assembly body are looked upon as the elements of polychromatic sets, the blocking relationships between the two parts are considered as the holistic contours of polychromatic sets, the possible displacement model is built. The locating datum model and the possible displacement model constitute the assembly relation model of polychromatic sets. The locating datum equation and possible displacement can be gained from the locating datum model and the possible displacement model according to the algorithms, so all questions in the assembling process only on the conceptions of logic and set $[19,20]$. The method has been used in car assembly $[21,22]$.

\subsection{Application of the polychromatic sets in the work flow modeling}

Directional graph and Petri net are the two-workflow Modeling technique used generally. The process activity and state are represented using nodes of directional graph; the mutual relationships of the nodes are represented with the directional arc of directional graph. The directional graph is difficult for the complicated business process modeling. Petri net is a kind of graphical and formal modeling tool and is suitable for describing system with concurrency and asynchrony. However, it has some weaknesses for modeling business process: (1) The business process defined by Petri net is difficult to be understood by a non-specialist; (2) Petri net is inconvenient to represent data flow. We study the work flow modeling by using polychromatic sets, firstly the process nodes of the work flow are represented, and then model the work flow by using polychromatic sets on the foundation of the conjunction graph of the work flow nodes, the algorithms of work flow function path is proposed finally $[23-25]$.

\subsection{Application of the polychromatic sets in tolerance modeling}

The tolerance plays important role on the selection of manufacturing processes and assembly strategies. The current CAD system is only an entity constructor which provides limited support for tolerance capabilities reflecting as the limitation of contacting the tolerance with the corresponding geometric entities: the size tolerance with dimension, form tolerances with form and locating tolerances with orientation. In order to add the tolerance function into the current $\mathrm{CAD}$ system, the tolerance representation model should be built firstly, in which all types of tolerance can be organized and represented in the independent way. The tolerance representation 
model not only represents the semanteme difference for different types tolerances, but also represents the rock-frame as the carrier for the tolerance: geometric entities and metric relations. A kind of hierarchical tolerance representation model based on feature is proposed by Professor Yusheng in Zhejiang University. We represented the hierarchical rock-bottom frame of this model by using polychromatic sets, and the reason of the $\mathrm{CP}$ layer is achieved which provided the foundation for the programming for the tolerance model [26]

\section{Conclusion}

Our team supervised by professor Zongbin $\mathrm{Li}$ has apply polychromatic sets into enterprises, including the product Conceptual design modeling, product assembly planning modeling, workflow modeling and Tolerance Information modeling. In the polychromatic sets all enterprise information are considered into the Boolean matrix, so it has excellences, such as high formalization, facilitated to programme and complexity simpleness etc, comparing with the traditional modeling tools. In the actual application use the polychromatic sets to organize the information of hierarchy model, so the polychromatic set have wide application foreground in the modeling and analyzing the complicated system and networks.

\section{References}

1. C.S. Leem and S. Kim, Introduction to an Integrated Methodology for Development and Implementation of Enterprise Information Systems, The Journal of Systems and Software 60, 249-261(2002).

2. V.V. Pavlov, Polychromatic Graph of Mathematics Simulation for Technical System, In the Proceedings of Scientific and Technical Conference (CAD-88), (Plovdiv, 1988), pp.8-10.

3. V.V. Pavlov, Mathematics Simulation of Discrete Production System, Information Technology 10, 15-19 (1995).

4. V.V. Pavlov, Polychromatic Sets and Graghs for CALS (STANKIN Press, Moscow, 2002).

5. Z. Li and L. Xu, Polychromatic sets and its application in simulating complex objects and systems, Computers and Operations Research 30(6), 851-860 (2003).

6. Z. Li, Research and Application of Polychromatic Sets in Advanced Manufacturing (China Waterpower Press and Knowledge Press, Beijing, 2005).

7. Z. Li, L. Zhao, Y. Ling, and T. Li, Study of Polychromatic Sets and Its Application in the Concept Design for Mechanical Products, Journal of Computer-Aided Design \& Computer Graphics 7, 688-692(2002).

8. Y. Tang, Study on the Conceptual Design Product Information Modeling and Schemes Reasoning Technology Based on Polychromatic Sets Theory, Xi'an Jiaotong University Thesis, 2003 (unpublished), p. 60.

9. H. Song and Z. Lin, Formalization of Conceptual Design Product Model Based on Polychromatic Sets, Journal of System Simulation 13, 298-306 (2001). 
10. F. Tang, Study of the Conceptual Design for Mechanical Products Based on Polychromatic Sets Theory and the Arithmetic of Design Candidate Identification, Xi'an Jiaotong University Thesis, 2002 (unpublished), p. 57.

11. Y. Tang, Z. Li, and S. Li, Formalized Solution of Conceptual Design Based on Polychromatic Sets, Journal of Xi'an Jiaotong University 38(1), 68-72 (2004).

12. W. Liu, Z. Li, and S. Li, Conceptual Design of Industrial Manipulator Based on Polychromatic Sets, China Mechanical Engineering 14, 158-162 (2003).

13. S. Li and Z. Li, An Evaluation Method of Conceptual Design Alternation for Machining Center, Machine Design and Research 12(5), 13-21 (2003).

14. $\mathrm{S}$. $\mathrm{Li}$ and $\mathrm{Z}$. Li, Conceptual Design Alternatives for Machining Center Based on Polychromatic Sets, Journal of Mechanical Engineering 40(6), 118-122 (2004).

15. L. Xu, Z. Li, S. Li, and F. Tang, A Polychromatic Sets Approach to Conceptual Design of Machine Tools, International Journal of Production Research 43(12), 2397-2421 (2005).

16. X. Gao and Z, Li, Study on Modeling and Reasoning Technology of Conceptual Design Based on Polychromatic Sets, China Mechanical Engineering (To appear).

17. X. Gao and Z. Li, A Polychromatic Sets Approach to Mechanism Synthesis, In the Proceedings of the International Conference on Information Technology in Science, Education and Industry (ISEI-2005), edited by A.T. Gupiev (Arhangelick, 2005), pp.228-235.

18. C. Li, Study and Development of an ASP System Based on Polychromatic Sets. Xi'an Jiaotong University Thesis, 2002 (unpublished), p. 57.

19. H. Zhang, Research on the Algorithm of Using Polychromatic Sets to Assembly Sequence Generation in Product, Xi' an Jiaotong University Thesis, 2004 (unpublished), p. 69.

20. B. Zhang, H. Zhang, S. Zhao, and Z. Li, Product Assembly Planning Modeling and Algorithm Based on Polychromatic Sets , Journal of Xi'an Jiaotong University 39(11), 12541258 (2005).

21. C Zhou, Research of Car Body Assembly Planning Model and Algorithm Based Polychromatic Sets, Xi'an Jiaotong University Thesis, 2005 (unpublished), p. 68.

22. S. Zhao and Z. Li, Non-forced Decomposition and Assembly Sequences Algorithm of Car Body, Xi'an Jiaotong University Research Report, 2006 (unpublished), p. 26.

23. X. Gao and Z. Li, Workflow Modeling Method Based on UML and Polychromatic Sets, Computer Integrated Manufacturing System-CIMS (To appear).

24. J. Zhu and Z. Li, Modeling and Route Acquired Algorithm of Workflow Using Polychromatic Sets, Journal of Xi'an Jiaotong University (To appear).

25. X. Gao and Z, Li, Formalizing UML Activity Diagram-Polychromatic Sets Approach, In the Proceedings of the International Conference on Computer Science for Design and Technology (CSDT-2005), edited by U.M. Colomentsev (Moscow, 2005), pp. 221-224.

26. B. Zhang and Z. Li, Study on Modeling of Tolerance Information and Reasoning Technique Study Using Polychromatic Sets, Journal of Mechanical Engineering 41(10), 111$116(2005)$. 